\title{
Fate of the Fc fusion protein aflibercept in retinal endothelial cells: competition of recycling and degradation
}

\author{
Heidrun L. Deissler ${ }^{1}$ (D) $\cdot$ Gerhard K. Lang ${ }^{1} \cdot$ Gabriele E. Lang $^{1}$ \\ Received: 19 January 2018 / Revised: 1 October 2018 / Accepted: 16 October 2018 / Published online: 26 October 2018 \\ (C) The Author(s) 2018
}

\begin{abstract}
Purpose Intravitreal injection of the VEGF-binding protein aflibercept is widely used to treat various ocular diseases. In vitro, immortalized bovine retinal endothelial cells (iBREC) take up and transport aflibercept through the cell layer in a serumdependent manner, likely mediated through the neonatal $\mathrm{Fc}$ receptor $(\mathrm{FcRn})$, but degradation of the $\mathrm{Fc}$ domain-containing protein might be a competing intracellular process. Therefore, aflibercept's associations with proteins either involved in FcRn-mediated transport or in the lysosomal pathway were studied.

Methods Confluent iBREC pre-cultivated with or without FBS were exposed for $4 \mathrm{~h}$ to in vivo achievable $250 \mu \mathrm{g} / \mathrm{ml}$ aflibercept, before cells were harvested for immunofluorescence staining or preparation of protein extracts. Intracellular localization of aflibercept and putative co-localizations with proteins involved in transport of IgG/FcRn complexes, i.e., endosomal Rab4 and Rab11, components of the cytoskeleton, motor proteins, or with marker proteins characteristic of multivesicular bodies or lysosomes were assessed by co-immunofluorescence stainings. Amounts of expressed endogenous proteins and of internalized aflibercept were determined by Western blot analyses.

Results Aflibercept-specific perinuclear staining overlapped with that of the motor protein dynein whereas double staining with an anti-kinesin antibody resulted in a patchy pattern. In addition, aflibercept was typically present close to microtubules and often co-localized with $\alpha$-tubulin. Rab4 and Rab11 stainings partly overlapped with the perinuclear staining of aflibercept whereas colocalization with Rab7 (in late endosomes/lysosomes) was only rarely seen. Interestingly, aflibercept but not the IgG bevacizumab broadly co-localized with the cation-independent mannose 6-phosphate receptor characteristic of multivesicular endosomes. In accordance with partial degradation beside transcytosis, the amount of intracellular aflibercept increased when cells were treated with protease inhibitors MG-132 or MG-101. Serum-deprived iBREC expressed less Rab11 and dynein but slightly more Rab4.

Conclusion After uptake by iBREC, aflibercept is present in organelles associated with FcRn-mediated transport, but part of the protein is subject to degradation. Transport inhibition of aflibercept during cultivation without FBS is likely a consequence of an attenuated exocytosis due to decreased expression of Rab11.
\end{abstract}

Keywords Retinal endothelial cells $\cdot$ Aflibercept $\cdot$ Fc fusion protein $\cdot \operatorname{IgG} \cdot$ Neonatal Fc receptor $\cdot$ Recycling $\cdot$ Degradation

\section{Introduction}

Aflibercept, a recombinant protein binding to members of the family of vascular endothelial growth factors (VEGFs), is widely used to treat various ocular diseases associated with

Heidrun L. Deissler

heidrun.deissler@uniklinik-ulm.de

1 Department of Ophthalmology, University Hospital of Ulm, Prittwitzstrasse 43, 89075 Ulm, Germany an elevated VEGF expression [1,2]. After intravitreal injection into the mammalian eye, aflibercept, which contains an Fc (fragment crystallizable) domain, was detected in ocular structures including retinal vessels and the retinal pigment epithelium (RPE), implying at least partial clearance of aflibercept via the posterior route [3-5]. Accordingly, retinal endothelial cells (REC) or cells of the RPE take up aflibercept in vitro, and transcellular transport through these types of cells was observed [6-8]. A carrier protein likely involved in shuttling IgG and other Fc-containing polypeptides is the neonatal $\mathrm{Fc}$ receptor/transporter $(\mathrm{FcRn})$, which indeed is expressed in various parts of the eye including the retinal endothelium and 
cultivated REC [9-12]. In human dermal microvascular endothelial cells (HMEC), the FcRn is present in early endosomes characterized by presence of early endosome antigen 1 (EEA1) and the small Ras-like GTPase 4 (Rab4) [13-15]. In these organelles, it binds at low $\mathrm{pH}$ to $\mathrm{IgG}$ that has been internalized by pinocytosis, after which the complex is separated from unbound $\operatorname{IgG}$ and other proteins in sorting endosomes (positive for Rab4 and Rab11) [9, 13-15]. FcRnbound IgG is then transported by recycling exosomes (positive for Rab11 but not for Rab4) to be released at the cell surface, thereby avoiding subsequent intracellular degradation in lysosomes [13, 15, 16]. In contrast, unbound IgG or other proteins destined to be eventually degraded in lysosomes, could be detected at an earlier stage mainly in multivesicular endosomes characterized by the presence of the cationindependent mannose 6-phosphate receptor (CI-M6PR) [17].

Because aflibercept is a protein with an Fc terminus of a human IgG1, a role of the FcRn in its transport through REC is a plausible assumption, recently supported by our investigations with immortalized bovine REC (iBREC). Through a monolayer of these cells, aflibercept is transported via an FcRn-mediated process, which was also observed in similar experiments with the VEGF-A-binding humanized IgG bevacizumab [7, 12]. However, aflibercept as an Fc fusion protein has distinct properties different from those of a classical IgG and this might result in at least partly altered mechanisms of intra-REC transport. Fc fusion proteins indeed tend to have a shorter serum half-life compared with IgG often due to a lower binding affinity to the FcRn $[18,19]$. Therefore, we investigated whether aflibercept taken up by iBREC was co- localized with proteins involved in an FcRn-mediated transport or those indicative of the lysosomal route. The human and bovine homologs of proteins likely participating in the investigated processes, i.e., FcRn, Ras-like GTPases, CI-M6PR, and others, are highly conserved or even identical, allowing experiments based on the well-established model of iBREC and double immunofluorescence staining with available specific antibodies $[20,21]$. It was also directly confirmed in several studies that human IgG is efficiently bound, transported, and released by bovine FcRn in vivo and in vitro [22-24].

\section{Material and methods}

\section{Aflibercept and antibodies}

Bayer Vital GmbH (Leverkusen, Germany) kindly provided Eylea $(40 \mathrm{mg} / \mathrm{ml}$ aflibercept in $10 \mathrm{mM}$ sodium phosphate, $40 \mathrm{mM} \mathrm{NaCl}, 0.03 \%$ polysorbate $20,5 \%$ sucrose, $\mathrm{pH} 6.2$ ) $[1,2]$. The humanized anti-VEGF antibody bevacizumab (Avastin; $25 \mathrm{mg} / \mathrm{ml}$ in $50 \mathrm{mM}$ sodium phosphate, $6 \% \alpha, \alpha$ trehalose dihydrate, $0.04 \%$ polysorbate $20, \mathrm{pH} 6.2$; Roche Pharma, Grenzach-Wyhlen, Germany) was repackaged at the pharmacy of the University Hospital Ulm and provided in syringes, which were stored at $4{ }^{\circ} \mathrm{C}$ not longer than 4 weeks [25]. Tables 1 and 2 provide all relevant information on primary and secondary antibodies used for immunofluorescence staining or Western blot analyses.

Table 1 Primary antibodies used

\begin{tabular}{|c|c|c|c|c|}
\hline Target & Host & Type & Source & Working concentrations \\
\hline Actin & Mouse & Monoclonal & Clone AC-40, Abcam (Cambridge, UK), ab11003 & $\mathrm{WB}, 500 \mathrm{ng} / \mathrm{ml}$ \\
\hline Caveolin-1 & Rabbit & Polyclonal & Abcam, ab2910 & $\mathrm{WB}, 20 \mathrm{ng} / \mathrm{ml} ; \mathrm{IF}, 1 \mu \mathrm{g} / \mathrm{ml}$ \\
\hline CI-M6PR/IGF2-R & Mouse & Monoclonal & Thermo Fisher Scientific (Langenselbold, Germany), MA1-066 & $\mathrm{IF}, 2 \mu \mathrm{g} / \mathrm{ml}$ \\
\hline Claudin-1 & Rabbit & Polyclonal & JAY.8, Thermo Fisher Scientific, 51-9000 & $\mathrm{WB}, 1 \mu \mathrm{g} / \mathrm{ml}$ \\
\hline Claudin-5 & Rabbit & Polyclonal & Thermo Fisher Scientific, 34-1600 & $\mathrm{WB}, 100 \mathrm{ng} / \mathrm{ml}$ \\
\hline $\begin{array}{l}\text { Dynein } \\
\quad \text { (intermediate chain } 1, \text { IC74) }\end{array}$ & Mouse & Monoclonal & Clone 74.1, Abcam, ab23905 & $\mathrm{WB}, 1 \mu \mathrm{g} / \mathrm{ml} ; \mathrm{IF}, 10 \mu \mathrm{g} / \mathrm{ml}$ \\
\hline EEA1 & Rabbit & Polyclonal & Abcam, ab137403 & $\mathrm{WB}, 70 \mathrm{ng} / \mathrm{ml}$; IF, $20 \mu \mathrm{g} / \mathrm{ml}$ \\
\hline FcRn (large chain) & Rabbit & Polyclonal & Bio-techne (Wiesbaden, Germany), NBP1-89127 & $\mathrm{WB}, 3 \mu \mathrm{g} / \mathrm{ml}$ \\
\hline Kinesin (heavy chain) & Mouse & Monoclonal & Clone SUK-4, Abcam, ab28060 & IF, $1.3 \mu \mathrm{g} / \mathrm{ml}$ \\
\hline Rab4 & Rabbit & Polyclonal & Thermo Fisher Scientific, PA3-912 & WB, 1:500; IF, 1:75 \\
\hline Rab7 & Rabbit & Polyclonal & Thermo Fisher Scientific, PA5-22959 & IF, $1: 100$ \\
\hline Rab11a, Rab11b, Rab11c & Goat & Polyclonal & Sicgen Antibodies (Carcavelos, Portugal), AB3035 & $\mathrm{WB}, 1.5 \mu \mathrm{g} / \mathrm{ml}$ \\
\hline Rab11 & Rabbit & Polyclonal & Abcam, ab3612 & IF, $5 \mu \mathrm{g} / \mathrm{ml}$ \\
\hline$\alpha$-Tubulin & Mouse & Monoclonal & Clone DM1A, Abcam, ab7291 & $\mathrm{WB}, 200 \mathrm{ng} / \mathrm{ml}$; IF, $1 \mu \mathrm{g} / \mathrm{ml}$ \\
\hline Vimentin & Mouse & Monoclonal & Clone RV202, Abcam, ab8978 & $\mathrm{WB}, 70 \mathrm{ng} / \mathrm{ml}$; IF, $1 \mu \mathrm{g} / \mathrm{ml}$ \\
\hline
\end{tabular}

$W B$, Western blot analyses; $I F$, immunofluorescence stainings; $C I-M 6 P R / I G F$-2R, cation-independent mannose 6-phosphate receptor/insulin-like growth factor 2 receptor; $E E A 1$, early endosomal antigen 1 
Table 2 Secondary antibodies used

\begin{tabular}{llllll}
\hline Target & Host & Type & Conjugate & Source & Working concentrations \\
\hline IgG, $\gamma$-chain, human & Goat & Polyclonal & Coupled to HRP & Thermo Fisher Scientific, 628,420 & WB, 1:5000 \\
Whole IgG, rabbit & Goat & Polyclonal & Coupled to HRP & Biorad (Munich, Germany), 170-5046 & WB, 1:30,000 \\
Whole IgG, mouse & Goat & Polyclonal & Coupled to HRP & Biorad, 170-5047 & WB, 1:30,000 \\
IgG, H+L chains, goat & Donkey & Polyclonal & Coupled to HRP & Bio-techne, HAF109 & WB, 1:4000 \\
IgG, H+L chains, human & Goat & Polyclonal & Coupled to AlexaFluor594 & Thermo Fisher Scientific, A11014 & IF, 1:500 \\
IgG, H+L chains, rabbit & Goat & $\mathrm{F}\left(\mathrm{ab}^{\prime}\right)_{2}$ fragment & Coupled to AlexaFluor488 & Thermo Fisher Scientific, A11070 & IF, 1:500 \\
IgG, H+L chains, mouse & Goat & $\mathrm{F}\left(\mathrm{ab}^{\prime}\right)_{2}$ fragment & Coupled to AlexaFluor488 & Thermo Fisher Scientific, A11017 & IF, 1:500 \\
\hline
\end{tabular}

$H R P$, horseradish peroxidase; $W B$, Western blot analyses; $I F$, immunofluorescence stainings

\section{Treatment of iBREC with effectors}

Generation and characterization of telomerase-immortalized microvascular endothelial cells from bovine retina (iBREC), which even after extended cultivation show the cobblestone-like morphology typical of EC-forming retinal capillaries, were comprehensively described $[12,21,26]$. Cells were cultivated on fibronectin-coated (Corning, Amsterdam, The Netherlands) surfaces in Endothelial Cell Growth Medium MV (ECGM; Promocell, Heidelberg, Germany) containing $1 \mathrm{~g} / \mathrm{l}$ glucose, $0.4 \%$ Endothelial Cell Growth Supplement $/ \mathrm{H}, 90 \mu \mathrm{g} / \mathrm{ml}$ heparin, $10 \mathrm{ng} / \mathrm{ml}$ human epidermal growth factor (hEGF), $100 \mathrm{nM} 11 \beta$ hydroxycortisone, and 5\% fetal bovine serum (FBS) as described previously $[12,21]$. Cells were used from passages 25 to 60 counting from the stage of primary culture for which we had confirmed stable expression of marker proteins typical for EC (e.g., von Willebrand factor, tight junction-proteins claudin-1 and claudin-5) and proteins under investigation $[6,7,12,21]$. Pericytes or other cells expressing $\alpha$-smooth muscle actin are not present in iBREC cultures [21]. To ensure authenticity and optimal conditions of iBREC in the experiments, we routinely recorded their characteristic proliferation profile by electric cellsubstrate impedance measurements using the microelectronic biosensor system for cell-based assays xCELLigence RTCA DP (Acea, OLS, Bremen, Germany) [27]. All experimentsrepeated at least twice-were performed with confluent monolayers of iBREC formed after cultivation for 4 days. In control experiments, we processed the cells identically in medium only lacking the effector(s) investigated.

Prior to investigations with confluent iBREC, ECGM was replaced with SHM (same medium as ECGM but lacking hEGF) or - to study the effect of FBS - with SFM (same as SHM but without FBS) for 1 day. Aflibercept (or bevacizumab) was added for additional $4 \mathrm{~h}$ before cells were harvested for preparation and analyses of subcellular fractions or fixed for immunofluorescence staining (see below). VEGF-binding proteins were used at a final concentration of $250 \mu \mathrm{g} / \mathrm{ml}$, resembling the in vivo situation after intravitreal injection $[2,6,7,12]$.

To assess the effects of the protease inhibitors MG-101 or MG-132 (Selleckchem, Absource Diagnostics, Munich,
Germany) on barrier stability, confluent iBREC grown on gold electrodes (E-Plates 16 PET, Acea) were treated with these substances at concentrations ranging from $20 \mathrm{nM}$ to $5 \mu \mathrm{M}$ or to $20 \mu \mathrm{M}$, respectively [27-30]. The cell index as a measure of permeability was recorded every $5 \mathrm{~min}$ for at least $24 \mathrm{~h}$ [27].

\section{Preparation of protein extracts}

Fresh or frozen cell pellets were subjected to subsequently carried out extraction steps with different buffers (ProteoExtract Subcellular Proteome Extraction Kit, Merck Millipore, Darmstadt, Germany), resulting in subcellular fractions containing proteins localized in the cytoplasm, in membranes/organelles, or components of the cytoskeleton [7, 12]. Protein concentrations in the samples were determined using the Pierce Thermo Scientific BCA Protein Assay Kit according to the manufacturers' instructions.

\section{Western blot analyses and imaging}

For Western blot analyses, proteins ( $3 \mu \mathrm{g} / \mathrm{lane})$ were separated by SDS-polyacrylamide electrophoresis under reducing conditions followed by electroblotting as described in detail previously [7, 27]. Appropriate IgG-horseradish peroxidase conjugates (Table 2) and the chemiluminescence kit Pierce ECL Plus Western Blotting Substrate (Thermo Fisher Scientific) were used to detect antigen-bound primary antibodies. As previously observed, the horseradish peroxidase conjugate used to detect human IgG gave rise to an additional unspecific signal when isolated cytoskeleton proteins were analyzed $[6,7,12]$. Chemiluminescence signals from the so processed membranes were directly scanned with the imaging system Fusion Pulse TS (Vilbert Lourmat, VWR, Darmstadt, Germany) resulting in images with bright specific bands contrasting with a dark background. To quantify the signals, peak volumes of the corresponding bands $(\geq 4$ replicates) determined with EvolutionCapt software (Vilbert Lourmat) were standardized in relation to those obtained from similarly processed control cells. 


\section{Double immunofluorescence staining}

Confluent iBREC were cultivated on fibronectin-coated two-chamber slides ( $\mathrm{x}$-well PCA Tissue Culture Chambers; Sarstedt, Nuembrecht, Germany) and exposed to aflibercept or bevacizumab for $4 \mathrm{~h}$ as described above $[7,12]$. After methanol-fixation $\left(10 \mathrm{~min}\right.$ at $\left.-20{ }^{\circ} \mathrm{C}\right)$, cells were permeabilized for $10 \mathrm{~min}$ in $0.25 \%$ Nonidet P-40 substitute (Roche, Mannheim, Germany) diluted in PBS without $\mathrm{Ca}^{2+} / \mathrm{Mg}^{2+}$ ions (PBSd). After incubation of the slides in blocking solution (10\% ImmunoBlock, Roth, Karlsruhe, Germany) for $60 \mathrm{~min}$ at room temperature, they were treated for $30 \mathrm{~min}$ with AlexaFluor594conjugated antibodies binding to human IgG to detect aflibercept or bevacizumab. Slides were then exposed to primary antibodies recognizing endogenous proteins of interest for $1 \mathrm{~h}$ at room temperature, and subsequently to appropriate AlexaFluor488-conjugated goat $\mathrm{F}(\mathrm{ab})_{2}$ fragments for $30 \mathrm{~min}$. Primary and secondary antibodies were always diluted in $1 \%$ ImmunoBlock/PBSd. For examination by fluorescence microscopy (DM4000B, FW4000, Leica, Wetzlar, Germany), cells were embedded in ProLong Gold/Diamond Antifade Mountant with DAPI (Thermo Fisher Scientific) [7, 12, 27].

\section{Transcytosis assays}

Transcytosis assays were performed as published to assess transport of aflibercept through a confluent iBREC monolayer cultivated on membrane inserts $\left(4.7 \mathrm{~cm}^{2}\right.$, pore size $0.4 \mu \mathrm{m}$; Corning) from the lower to the upper chamber [7, 12]. After incubating iBREC for 1 day in SHM or SFM, aflibercept (final concentration, $250 \mu \mathrm{g} / \mathrm{ml}$ ) was placed in the bottom chamber and samples were then taken from the upper chamber at indicated time points. Presence of aflibercept in these samples was assessed by Western blot analyses and peak volumes of the corresponding bands (four replicates) determined with EvolutionCapt software (Vilbert Lourmat) were normalized in relation to those obtained from $1 \mathrm{ng}$ aflibercept.

\section{Statistical analyses}

Mann-Whitney tests or one-way analyses of variance (ANOVA) followed by Tukey's test were used to compare differing groups of quantified antigen-specific signals from Western blot analyses (Graph Pad Prism 6; Graph Pad Software, San Diego, USA), and differences resulting in $p$ values below 0.05 were considered significant. Means and standard deviations were provided as numbers or in scatter plots.

\section{Results}

\section{Aflibercept was present in organelles associated with FcRn-mediated transport and in multivesicular endosomes within $4 \mathrm{~h}$ after uptake}

To determine aflibercept's intracellular localization, we performed double-immunofluorescence stainings of iBREC treated with pharmacologically achievable $250 \mu \mathrm{g} / \mathrm{ml}$ of the therapeutic protein for $4 \mathrm{~h}$. Then aflibercept had been taken up and transported through an iBREC monolayer, and substantial amounts of the recombinant protein remained in the cells resulting in a pronounced perinuclear staining in addition to a faint and diffuse overall intracellular staining $[6,7]$. Direct targeting the FcRn was not possible because commercially available antibodies failed to give specific signals, but the assessed proteins were reported to be crucially involved either in the transport of FcRn/IgGcomplexes or in lysosomal processes in endothelial cells (EC) or other cell types [7, 11-17, 31-34].

Microtubule are involved in the transport of $\mathrm{IgG} / \mathrm{FcRn}$ complexes in various cell types [31-33]. Accordingly, aflibercept co-localized with the microtubule component $\alpha$ tubulin mainly in the perinuclear region close to or identical with the microtubule organization center (Fig. 1a, yellow arrows) but also close to the tubulin fibers in the periphery of the cells (Fig. 1a, blue arrows). Members of the motor protein family of dyneins transport cargo along microtubules from the plasma membrane to the cell body whereas kinesins are involved in similar transport in the opposite direction [33]. Interestingly, aflibercept-specific staining overlapped with that of the intermediate chain (IC74) of dynein in the perinuclear region (Fig. 1b) whereas signals specific for the heavy chain (HC) of kinesin were rather patchy with only a few overlaps (Fig. 1c, yellow arrows or arrowheads). Colocalization of aflibercept with other cytoskeletal proteins, e.g., actin or vimentin, or with caveolin-1-a component of caveolae - was not observed. In HMEC, early endosomes $(\rightarrow$ Rab4, EEA1), sorting endosomes ( $\rightarrow$ Rab4, Rab11), and recycling exosomes ( $\rightarrow$ Rab11) contain the FcRn or FcRn/ IgG complexes $[14,15]$. In accordance with our previous observation that the perinuclear staining of aflibercept often overlapped with that of EEA1, aflibercept was also co-localized in the perinuclear region with signals specific for Rab4 (Fig. 2a) [7]. In addition, stainings of aflibercept and Rab4 overlapped in the periphery of the cells. In the same subcellular regions, we also observed partial co-localization of aflibercept with Rab11 (Fig. 2b).

Internalized aflibercept might be degraded, and proteins destined for degradation were detected in multivesicular endosomes, characteristically containing CI-M6PR involved in the transport of hydrolases from the trans-Golgi network to lysosomes [17]. Remarkably, the immunofluorescence 
Fig. 1 Aflibercept is co-localized with $\alpha$-tubulin and dynein. Confluent iBREC treated with aflibercept were immunostained to detect aflibercept (red), $\alpha$ tubulin (a, green), dynein intermediate chain IC74 (b, green), or kinesin heavy chain (HC, $\mathbf{c}$, green). a $\alpha$-Tubulin fibers originating from the microtubule organization center stretched to the periphery of the cells. Aflibercept-specific signals overlapped with those of $\alpha$ tubulin at the microtubule organization center (yellow arrows). They were also close to the $\alpha$-tubulin fibers in the cell periphery (blue arrows). b Dynein signals around the nucleus overlapped with those of aflibercept (yellow arrows). c The strong perinuclear kinesinspecific staining and that of aflibercept gave rise to a mosaic pattern (yellow arrowheads). Scale bar, $10 \mu \mathrm{m}$

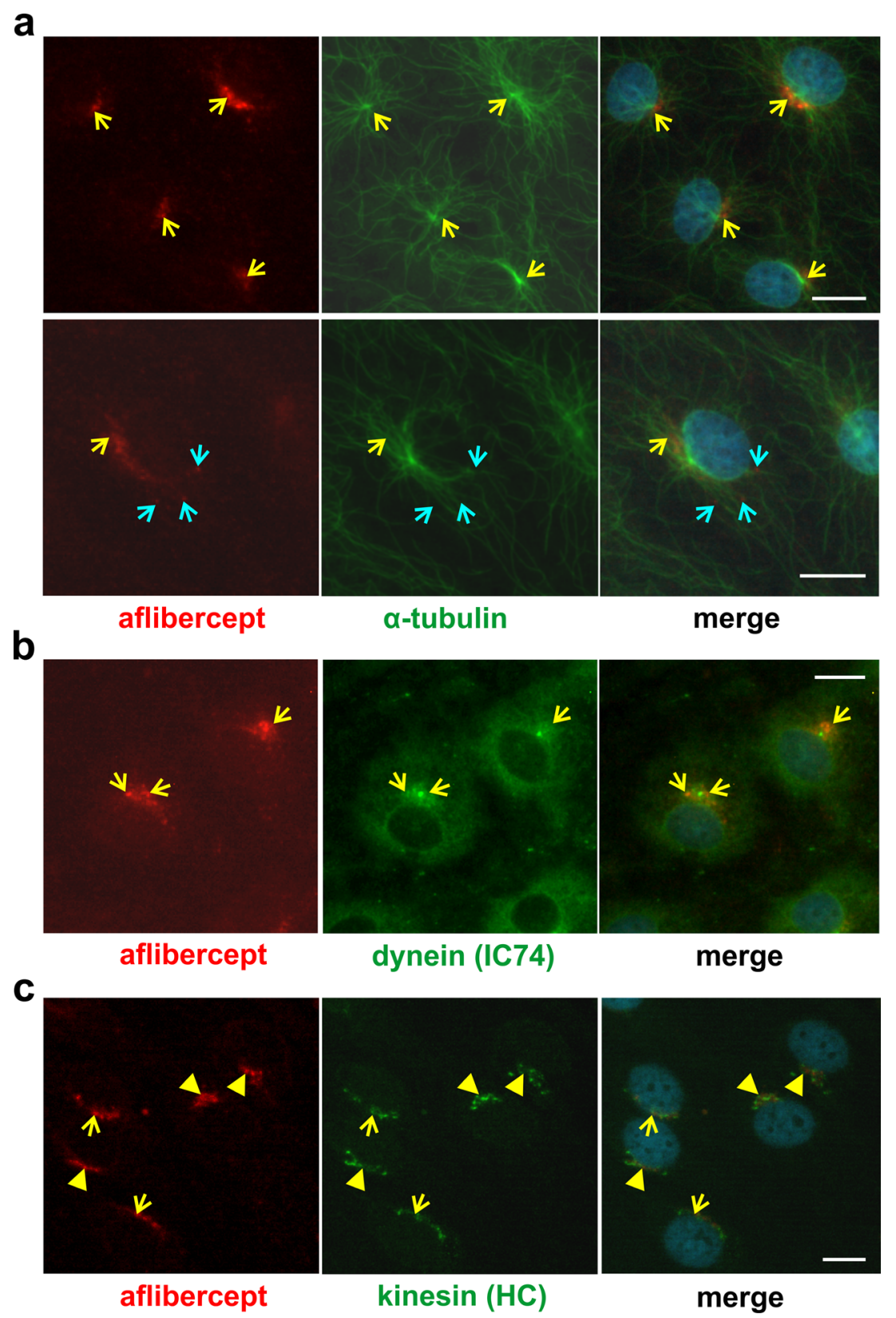

a

b

aflibercept

aflibercept kinesin (HC)

merge

stainings of aflibercept and CI-M6PR observed in the cells' perinuclear regions widely overlapped (Fig. 3a). Colocalization of aflibercept with Rab7, a marker of late endosomes and lysosomes in EC, was seen sporadically and then as patchy signals rather than mixed colors (Fig. 3b) [34]. To rule out that presence of aflibercept in multivesicular endosomes might be the unlikely result of species incompatibility due to the interaction between the human Fc portion and bovine $\mathrm{FcRn}$, we performed similar experiments with the VEGF-binding antibody bevacizumab also containing a human Fc terminus. As shown in Fig. 4 (yellow arrowheads or arrows), the immunofluorescence stainings specific for CIM6PR and bevacizumab barely overlapped.

\section{Inhibition of proteasomal and lysosomal proteases increased the amount of intracellular aflibercept}

Our data support the hypothesis that the internalized aflibercept is transported through an iBREC monolayer in complex with FcRn, but part of the recombinant protein may be degraded. The cysteine protease inhibitor MG-101 counteracts the activity of lysosomal cathepsins $\mathrm{L}$ and $\mathrm{B}$ at a concentration of $20 \mathrm{nM}$ or that of the non-lysosomal calpains I and II at $500 \mathrm{nM}$, respectively $[28,29]$. Exposure of iBREC to either effective concentration of MG-101 together with aflibercept for $4 \mathrm{~h}$ resulted in a slight but significant increase of aflibercept isolated collectively with proteins from membranes and organelles (Fig. 5a). 
Fig. 2 Aflibercept is co-localized with Rab4 and Rab11.

Aflibercept (red), Rab4 (a, green), or Rab11 (b, green) were

visualized by double

immunofluorescence staining after exposure of iBREC to the $\mathrm{Fc}$ fusion protein. Antibodies specific for Rab4 (a) or Rab11 (b) bound to a prominent perinuclear region stretching into the periphery of the cell with similar but not identical patterns. Overlapping of signals (yellow arrows) from Rab4 (a) or Rab11 (b) staining with those specific for aflibercept is most evident in the perinuclear region and partially seen in the peripheral areas. Scale bar, $10 \mu \mathrm{m}$
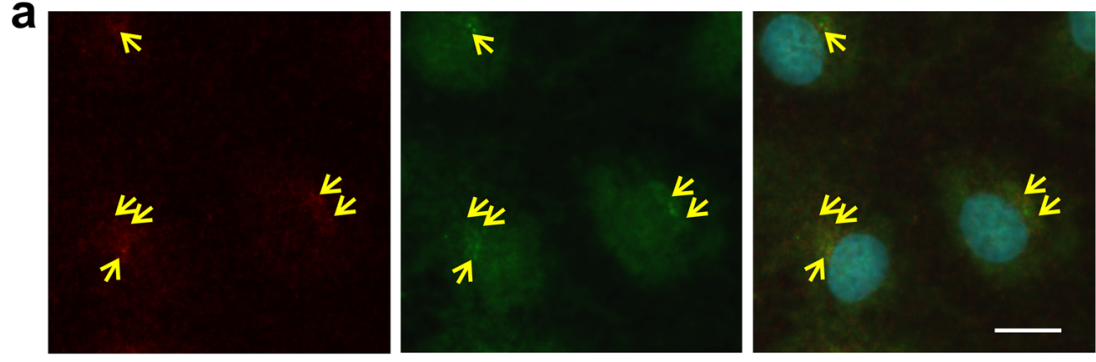

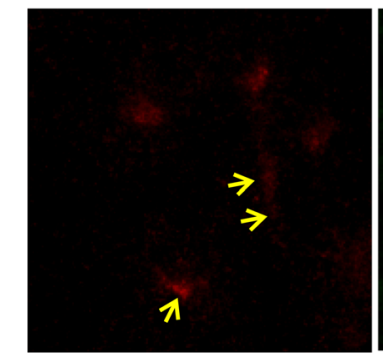

aflibercept

b

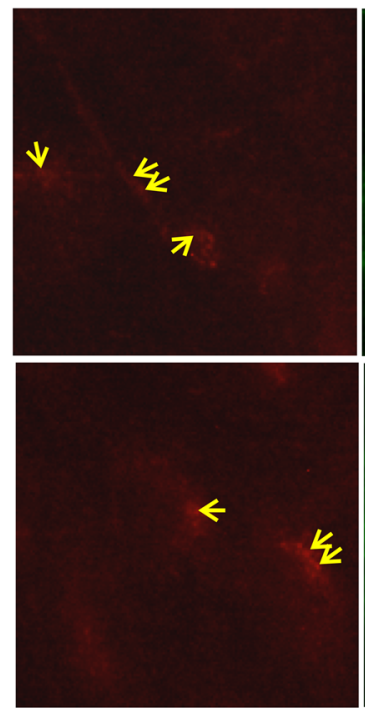

aflibercept

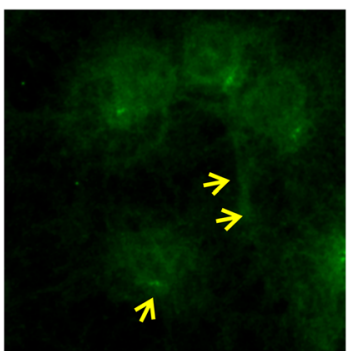

Rab4
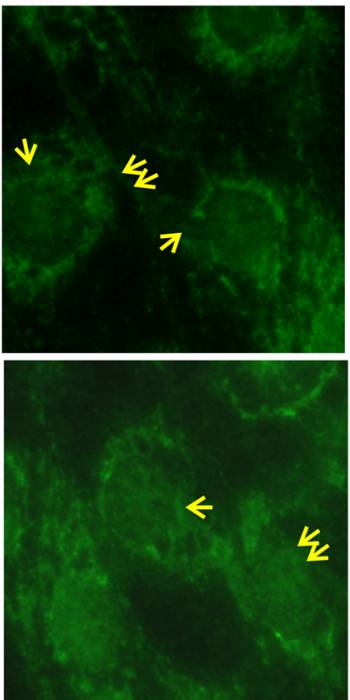

Rab11

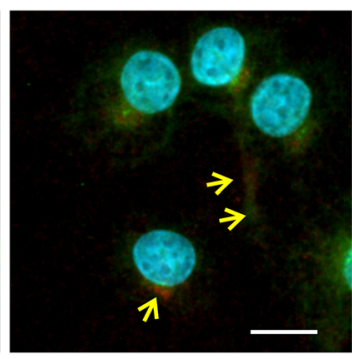

merge
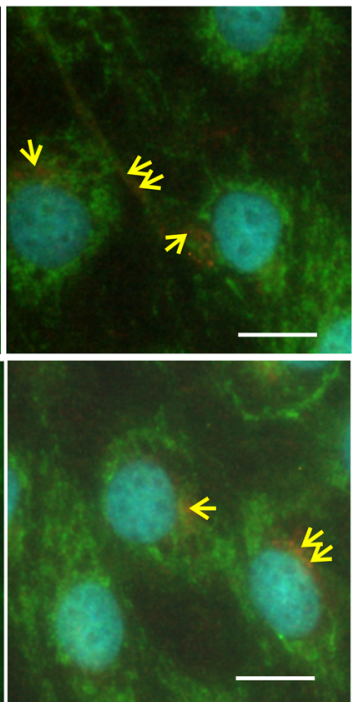

merge
Because most of the intracellular protein destined to be degraded enters the ubiquitin-proteasome pathway, we pre-treated iBREC with $20 \mathrm{nM}$ of the inhibitor MG-132 of proteasomal

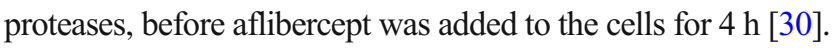
Western blot analyses of proteins subsequently isolated from the membranes/organelles and the cytoskeleton revealed that more aflibercept accumulated during protease inhibition (Fig. $5 b)$. As was to be expected, the amounts of endogenous proteins actin and claudin-5 were higher in samples isolated from cells treated with the inhibitors MG-101 or MG-132, confirming general inhibition of protein degradation pathways under these conditions (Fig. 5c, d). Higher concentrations of MG-101 or MG-132 could not be used because they resulted in barrier dysfunction and death of iBREC as recognized by cell index measurements.

\section{Serum depletion resulted in decreased expression of dynein and Rab11}

Aflibercept's transport through an iBREC monolayer was considerably slower when cells were cultivated without FBS and this coincided with a significantly increased amount of the recombinant protein retained in the cells (Fig. 6a, b) [7]. We also determined the cell index as a measure of iBREC permeability and found that it was high and in the same range when iBREC were cultivated with or without FBS. This confirms the presence of a stable barrier preventing paracellular transport and allowing for transcellular transport only. Based on the assumption that aflibercept's intracellular transport could be restricted as a consequence of a changed expression of involved proteins under these conditions, we analyzed their presence in iBREC 
Fig. 3 Aflibercept is largely colocalized with CI-M6PR. iBREC exposed to aflibercept were immunostained to detect aflibercept (red) and either the marker of multivesicular endosomes CI-M6PR (a, green) or Rab7 (b, green), specific for late endosomes and lysosomes. a The prominent CI-M6PR-specific staining close to the nucleus broadly overlapped with that of aflibercept (yellow arrows). b In contrast, aflibercept-specific signals and those specific for $\mathrm{Rab} 7$ gave rise to a mosaic pattern with few overlaps (yellow arrowheads). Scale bar, $10 \mu \mathrm{m}$ a

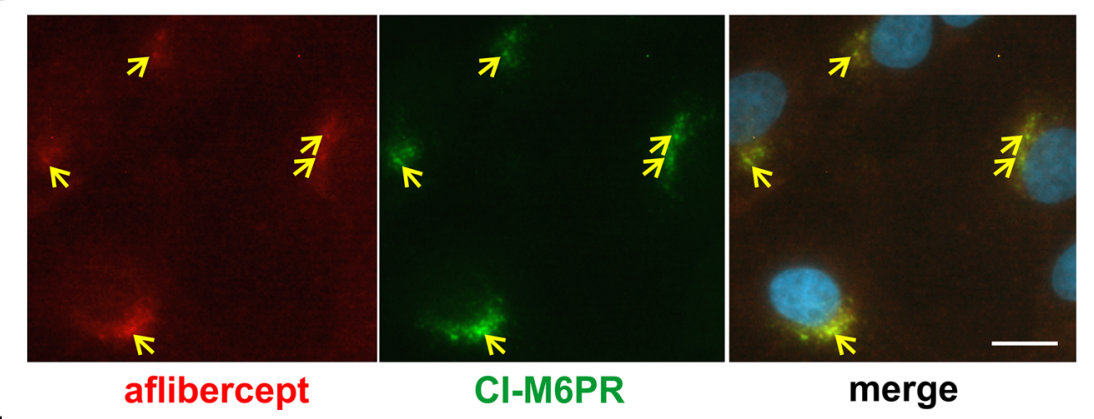

b

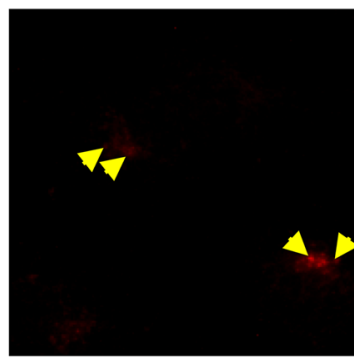

aflibercept

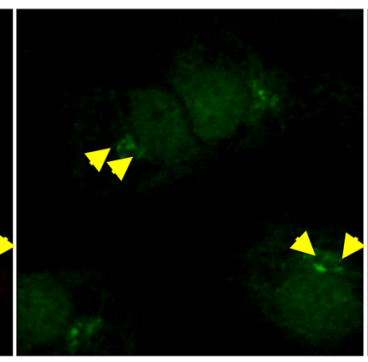

Rab7

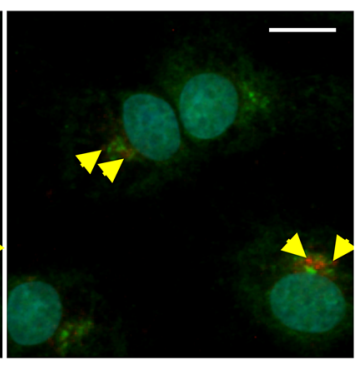

merge treated for $4 \mathrm{~h}$ with the Fc fusion protein after having been cultivated with or without FBS for 1 day. Western blot analyses of proteins from the cytoplasm, membranes, or organelles and the cytoskeleton showed that presence of aflibercept did not significantly affect the levels of investigated proteins at either condition. Reduced expression of the FcRn after cultivation of iBREC under serum-free conditions was not due to a general decline in protein expression as some proteins investigated were not changed and others were even expressed significantly stronger, e.g., tight junction-proteins claudin-1 and claudin-5 (Fig. 6b). Interestingly, the levels of Rab11 and dynein were also lower whereas that of Rab4 was higher after cultivation of iBREC without FBS (Fig. 6b). In addition, cultivation conditions resulted in changes of the subcellular localization of Rab4 and to a lesser extent of Rab11. Both proteins were more focused in the perinuclear region in cells cultivated without serum and overlapping of the perinuclear stainings of Rab4 and aflibercept was then slightly more pronounced.

\section{Discussion}

After its uptake by REC, aflibercept can be transported actively through the intracellular space but before its release from the cell surface at either side of the EC layer, it might enter the degradation pathway. That aflibercept has an Fc terminus suggests involvement of the FcRn in its transport and protection from proteolysis. To further elucidate aflibercept's fate inside iBREC, we performed double immunofluorescence stainings with marker proteins either associated with an FcRn-mediated transport ( $\rightarrow$ EEA1, Rab4, Rab11) or with the lysosomal pathway in EC ( $\rightarrow$ CI-M6PR, Rab7) [13-17, 34]. We also included proteins of the cytoskeleton ( $\rightarrow \alpha$-tubulin, actin, vimentin) as well as motor proteins ( $\rightarrow$ dynein, kinesin) in our analyses because these might play a role in FcRn-mediated transport along microtubules [31-33]. Obtained data on potential colocalization help to reveal the fate of internalized aflibercept although these marker proteins do not specify a single

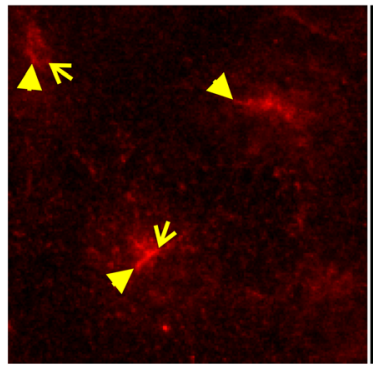

bevacizumab

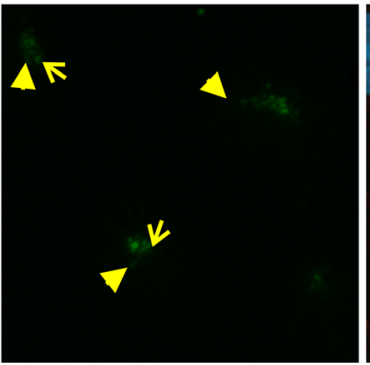

CI-M6PR

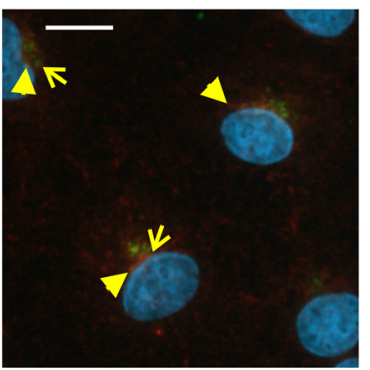

merge
Fig. 4 Bevacizumab is somewhat co-localized with CI-M6PR. After treatment of iBREC with the humanized IgG bevacizumab for $4 \mathrm{~h}$, cells were immunostained to visualize the IgG (red) or CI-M6PR (green). The prominent CI-M6PR-specific signals close to the nucleus only to a small extent overlapped with those of bevacizumab (yellow arrowheads). Scale bar, $10 \mu \mathrm{m}$ 


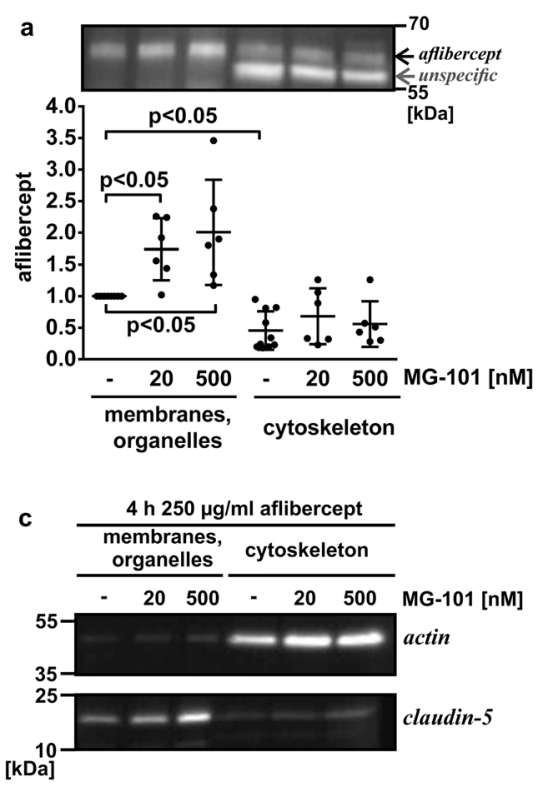

Fig. 5 Inhibition of lysosomal or proteasomal proteases resulted in accumulation of aflibercept. After exposure of iBREC to aflibercept with or without inhibitors of lysosomal (a, c MG-101) or proteasomal (b, d MG-132) proteases, cells were harvested for preparation of subcellular fractions. In these, aflibercept $(\mathbf{a}, \mathbf{b})$, actin, or claudin-5 (c, d) were determined by Western blot analyses. To quantify the afliberceptspecific signals, peak volumes of the corresponding bands were measured and set in relation to those obtained from cells not exposed to the inhibitors. a The amount of aflibercept isolated with proteins from organelles and membranes was significantly higher after treatment with

pathway or subcellular compartment. Although immunofluorescence stainings result in fixed images showing the situation at one time point, they have the advantage over live cell imaging that normal conditions rather than overexpressed recombinant proteins modified with fluorescence tags are analyzed. We chose to investigate aflibercept's localization in iBREC after its uptake in the absence of VEGF-A, because the tight barrier formed by the cells under these conditions only allowed transcellular transport without additional paracellular flow.

Aflibercept staining overlapped with or was close to that of $\alpha$-tubulin, suggesting an active transport along the microtubules. This assumption is supported by the also observed colocalization of aflibercept and the dynein intermediate chain, which is part of the dynein motor protein complex carrying cargo from the plasma membrane to the cell body. Colocalization of aflibercept with Rab4 and Rab11 as well as with CI-M6PR, evident after $4 \mathrm{~h}$ of exposure, pointed to the vesicular containers in which the protein is transported. Presence of aflibercept in vesicles containing Rab4 and/or Rab11 is in accordance with previously observed localization in EEA1-presenting early endosomes and supports the hypothesis that the FcRn plays an important role in its transcellular transport [7]. Remarkably, aflibercept also seemed to be present in multivesicular endosomes of iBREC indicated by its proximity to the CI-M6PR. Whereas CI-M6PR ${ }^{+}$
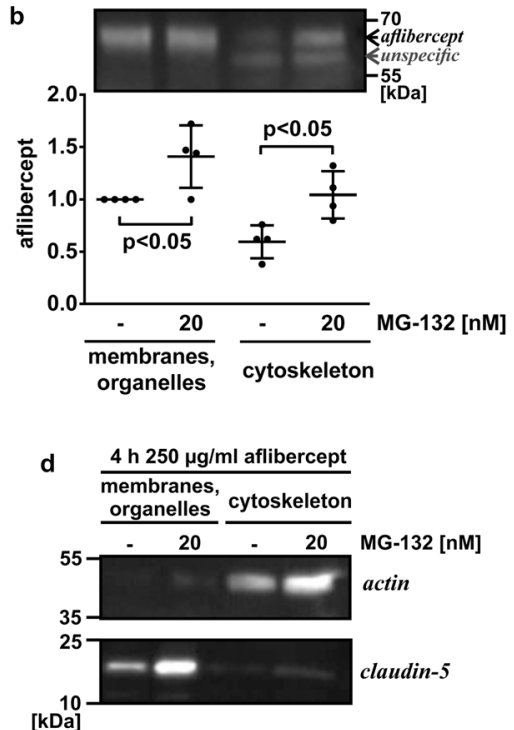

MG-101. b MG-132 also increased the amount of intracellular aflibercept. c, d Proteins from different subcellular compartments were efficiently separated without relevant cross-contamination: tight junctionprotein claudin-5 ( $\rightarrow$ plasma membrane) was almost exclusively isolated together with proteins from membranes and organelles, and actin was only present in the fraction containing cytoskeleton proteins. Stronger signals in the lanes with samples from cells treated with the inhibitors MG-132 and MG-101 are due to the general inhibition of intracellular protein degradation

multivesicular endosomes of HepG2 cells or of epithelial cells of the rat intestine contain the FcRn, it was not detected in such vesicles of HMEC [13, 32, 35]. However, a modified IgG not able to bind to the FcRn remained in sorting endosomes in HMEC, which then matured to multivesicular endosomes [13]. From this observation and our results, we conclude that unbound aflibercept molecules not interacting with the FcRn likely reach CI-M6PR ${ }^{+}$multivesicular endosomes. It is a reasonable assumption that this is not due to an impaired interaction between the human $\mathrm{Fc}$ terminus of aflibercept and the bovine FcRn because the bovine receptor efficiently transports human IgG through cells [7, 12, 22-24]. Accordingly, the humanized VEGF-binding IgG bevacizumab was only rarely detected in multivesicular endosomes in iBREC (see Fig. 4).

An increased amount of aflibercept internalized by iBREC as a consequence of general inhibition of lysosomal and proteasomal proteases showed that at least part of the $\mathrm{Fc} \mathrm{fu}-$ sion protein is indeed degraded. The observed partial overlap of aflibercept and Rab7 stainings supports this hypothesis as appearance of Rab7 indicates maturing of sorting endosomes to $\left(\operatorname{Rab} 7^{+}\right)$late endosomes, which transfer proteins destined for degradation to lysosomes by tubule-mediated processes [36]. Aflibercept's entering of a pathway leading to lysosomal degradation is in accordance with the reported lower serum half-lives of other Fc fusion proteins compared with 
a

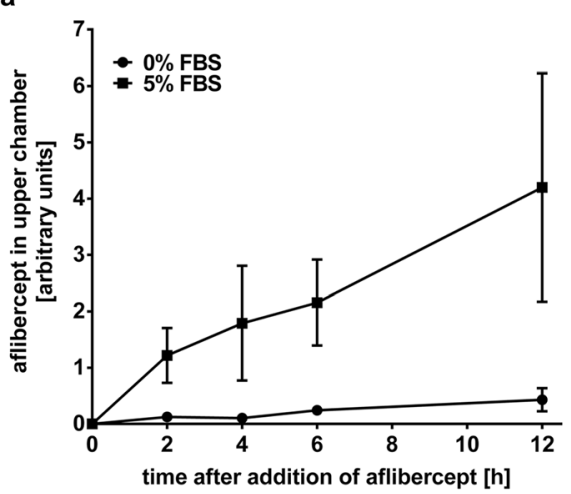

b

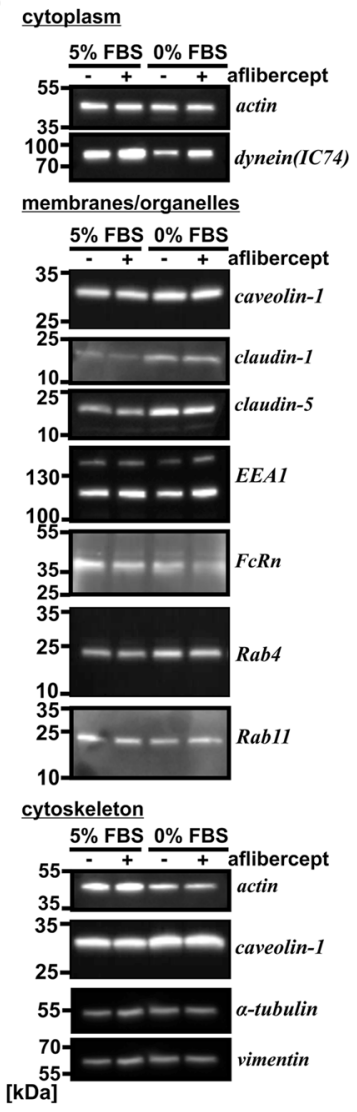

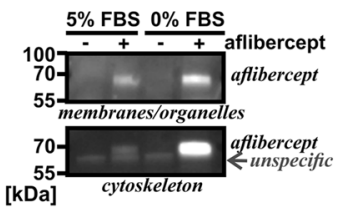

\begin{tabular}{lcc} 
& \multicolumn{2}{c}{ level at 0\% FBS } \\
\cline { 2 - 3 } & level at 5\% FBS & \\
\hline aflibercept (M/O) & $2.2 \pm 0.81$ & $\mathbf{p}<0.05$ \\
aflibercept (CS) & $21 \pm 12$ & $\mathbf{p}<0.05$ \\
\hline Fc-receptor & & \\
FcRn (large chain) & $0.44 \pm 0.09$ & $\mathbf{p}<0.05$ \\
\hline Endosomal proteins & & \\
EEA1 & $0.99 \pm 0.19$ & $\mathrm{p}>0.05$ \\
Rab4 & $1.59 \pm 0.33$ & $\mathbf{p}<0.05$ \\
Rab11 & $0.25 \pm 0.15$ & $\mathbf{p}<0.05$ \\
\hline
\end{tabular}

Transport and motor proteins

dynein (IC74) $\quad 0.46 \pm 0.08 \quad \mathbf{p}<\mathbf{0 . 0 5}$

$\alpha$-tubulin $\quad 1.29 \pm 0.71 \quad p>0.05$

caveolin-1 (M/O) $\quad 1.46 \pm 0.28 \quad \mathrm{p}<0.05$

caveolin-1 (CS) $\quad 1.42 \pm 0.18 \quad \mathbf{p}<0.05$

Structural proteins

actin (CP) $\quad 1.00 \pm 0.42 \quad p>0.05$

actin (CS) $\quad 0.68 \pm 0.16 \quad p<0.05$

vimentin $\quad 1.21 \pm 0.20 \quad p>0.05$

Tight-junction proteins

claudin-1 $\quad 1.47 \pm 0.13 \quad \mathrm{p}<0.05$

$\begin{array}{lll}\text { claudin-5 } & 2.25 \pm 0.44 \quad \mathrm{p}<0.05\end{array}$
Fig. 6 Cultivation of iBREC without FBS affected aflibercept's transport rate and intracellular amount, as well as expression of proteins potentially involved its transport. a iBREC monolayers grown on porous membrane inserts were exposed to culture medium with or without FBS for 1 day. Aflibercept was then added to the lower chamber and its presence was assessed and quantified by Western blot analyses as described in materials and methods in samples taken from the upper chamber. Transport of aflibercept through iBREC was significantly faster in the presence of
FBS. b After cultivation of iBREC with or without FBS for 1 day, aflibercept was added for additional $4 \mathrm{~h}$. Then cells were harvested for preparation of subcellular fractions as indicated. Relative expression levels of proteins were determined by Western blot analyses as described in materials and methods. Cultivation without FBS resulted in significantly more internalized aflibercept and decreased expression of Rab11 and dynein, whereas amounts of other proteins were higher. $\mathrm{M} / \mathrm{O}$, membranes/organelles; $\mathrm{CS}$, cytoskeleton; $\mathrm{CP}$, cytoplasm monoclonal antibodies, likely caused by lower affinity to the FcRn [18]. A serum half-live of aflibercept significantly lower than that of bevacizumab was also confirmed by experiments in which the proteins were administered to monkey eyes by intravitreal injection [19]. The intracellular stability of the VEGF-A-binding Fab fragment ranibizumab taken up by iBREC is even lower than that of aflibercept [37]. This might be due to the lack of an Fc terminus necessary for binding by the FcRn which is protective against intracellular proteolysis. Accordingly, a lower serum half-life after intravitreal injection was also observed [19].

The assumption that FcRn, Rab11, Rab4, and dynein all play important roles in aflibercept's transcellular transport is further supported by our observation that their expression or localization significantly changed when iBREC had been cultivated without FBS to slow down the transport process. Under all conditions investigated, iBREC established a strong barrier as confirmed by a high cell index, thereby allowing only transport through the cell layer. When iBREC were cultivated without FBS, the lower expression of FcRn, Rab11, and dynein was accompanied by an increased expression of other proteins including Rab4, and the accumulation of Rab4, Rab11, and EEA1 as well as aflibercept in the perinuclear region [7]. Interestingly, in HMEC sorting of an FcRn-IgG complex away from unbound $\operatorname{IgG}$ takes place in $\mathrm{Rab}^{+} /$ Rab $11^{+}$sorting endosomes which mature to $\mathrm{Rab}^{-} /$ Rab $11^{+}$recycling exosomes [15]. Considering these observations, decreased transport and accumulation of aflibercept in serum-deprived iBREC might be caused by shortage of Rab $11^{+}$recycling exosomes leading to attenuated exocytosis.

Studying the intracellular fate of $\operatorname{IgG}$ or related proteins like aflibercept in REC is of considerable importance because after their therapeutic intravitreal injection they quickly reach the pericytes and endothelial cells of retinal vessels. This was demonstrated for aflibercept, bevacizumab and Fc fragments, detected in retinal vessels 1 to 2 days after their intravitreal 


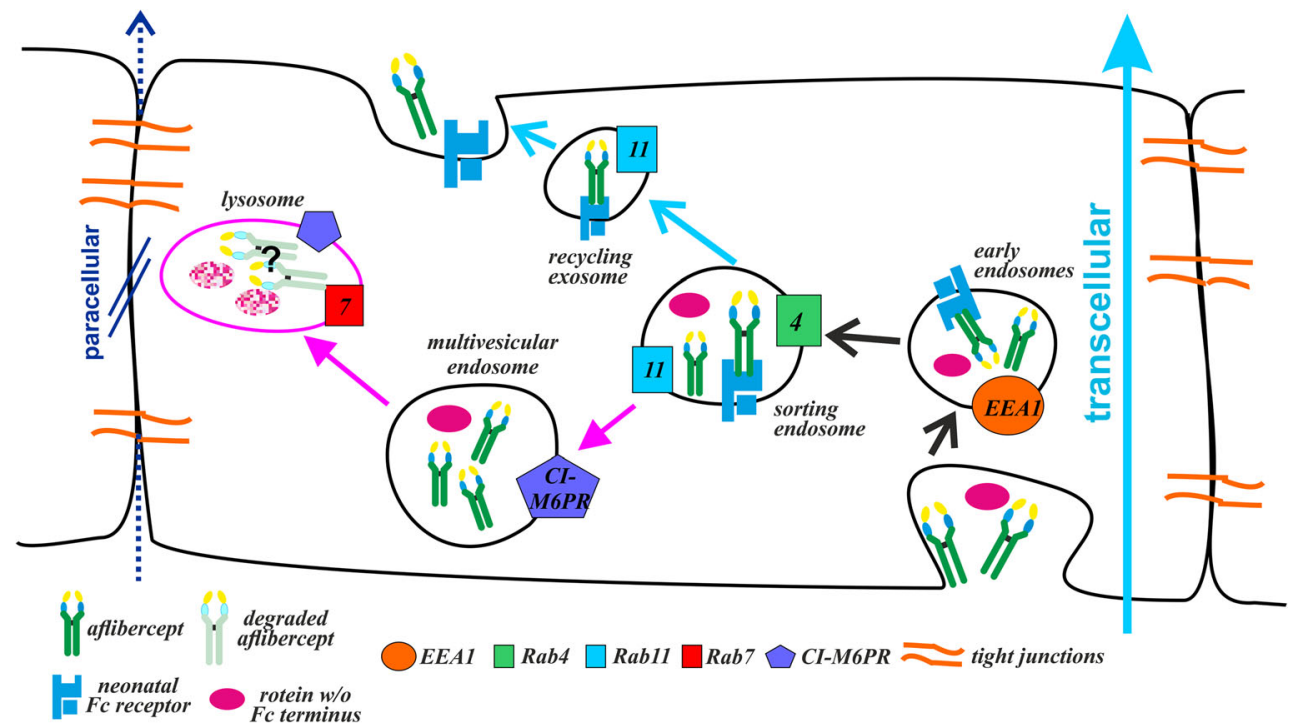

Fig. 7 Cartoon proposing the fate of aflibercept after its uptake by iBREC. Within a few hours of exposure, aflibercept is distributed to $\mathrm{EEA}^{+}$early endosomes, endosomes positive for Rab4 and/or Rab11, and CI-M6PR ${ }^{+}$multivesicular endosomes. This is in accordance with an FcRn-mediated transport of aflibercept through the intracellular space (light blue arrow), resulting in recycling of the $\mathrm{Fc}$ fusion protein

injection into the eyes of rodents and monkeys [3,38-41]. The role of the $\mathrm{Fc}$ domain has been emphasized by showing that a VEGF trap derivative, which instead of the Fc terminus contains a dimerized coiled-coil domain, has an increased half-life after intravitreal injection into the rabbit eye compared with the normal VEGF trap with an Fc region [4]. Together with the observation that after intravitreal injection bevacizumab was present in REC of wild-type mice but not in those of $\mathrm{FcRn}^{-1-}$ mice, these findings strongly support the hypothesis that the Fc domain primarily destines the fate of an intravitreally injected protein and that the FcRn plays a crucial role in this process [39]. An FcRn-dependent transport of aflibercept through cells of the RPE was also observed [8]. In these cells, aflibercept is co-localized with the motor protein myosin $7 \mathrm{a}$ and with proteins of the cytoskeleton ( $\rightarrow$ actin), indicating a similar though not identical behavior of the $\mathrm{Fc}$ fusion protein in the two different cell types forming the outer and inner blood retina barrier.

Summarizing, our results presented here are in accordance with the concept that aflibercept is transported through the intracellular space of REC in a process likely mediated by the FcRn as depicted in Fig. 7. Our previous findings that inhibition of the interaction between aflibercept and FcRn significantly slows down its transcellular transport also strongly support this hypothesis [7]. However, at least a fraction of the amount of internalized protein is subject to degradation, indicating that in detail the behavior of the Fc fusion protein inside REC is similar to though not identical with that of an IgG. at the surface of the cell. However, at least part of this protein is subject to degradation as indicated by our results on the effects of inhibitors of lysosomal or proteasomal proteases. Tight junctions closing the intercellular space prevent alternative paracellular transition (dark blue arrow) of the large aflibercept molecules

Acknowledgements The authors thank Edith Glass, Nadine Gubernath, Anita Ruepp, and Sarah Scislowski for expert technical assistance and Bayer Vital GmbH, Germany for a generous gift of aflibercept. The authors are grateful to Helmut Deissler, HD/U Ulm, Germany for his advice and for supporting the preparation of the manuscript.

Funding This study was supported through a research grant by Bayer Vital GmbH, Germany to Heidrun L. Deissler. The funding source did not have any influence on study design, data analyses and interpretation, writing of the manuscript, and decision on publishing.

\section{Compliance with ethical standards}

Conflict of interest Heidrun L. Deissler has received funding from Novartis Pharma GmbH, Bayer Vital GmbH, and lecture fees from Bayer Vital GmbH. Gerhard K. Lang has received honoraria from Bayer Vital GmbH. Gabriele E. Lang has received funding from Novartis Pharma GmbH, Bayer Vital GmbH, Boehringer Ingelheim Pharma, Allergan, and Alcon Pharma $\mathrm{GmbH}$ and has received honoraria as a consultant from Boehringer Ingelheim Pharma and as a consultant and speaker from Novartis Pharma GmbH, Carl Zeiss Meditec, Alimera Sciences, and Bayer Vital GmbH.

Ethical approval This article does not contain any studies with human participants or animals performed by any of the authors.

Open Access This article is distributed under the terms of the Creative Commons Attribution 4.0 International License (http:// creativecommons.org/licenses/by/4.0/), which permits unrestricted use, distribution, and reproduction in any medium, provided you give appropriate credit to the original author(s) and the source, provide a link to the Creative Commons license, and indicate if changes were made. 


\section{References}

1. Holash J, Davis S, Papadopoulos N et al (2002) VEGF-Trap: a VEGF blocker with potent antitumor effects. Proc Natl Acad Sci U S A 99:11393-11398. https://doi.org/10.1073/pnas.172398299

2. Do DV, Nguyen QD, Boyer D et al (2012) One-year outcomes of the DA VINCI study of VEGF Trap-eye in eyes with diabetic macular edema. Ophthalmology 119:1658-1665. https://doi.org/10. 1016/j.ophtha.2012.02.010

3. Julien S, Biesemeier A, Taubitz T, Schraermeyer U (2014) Different effects of intravitreally injected ranibizumab and aflibercept on retinal and choroidal tissues of monkey eyes. Br J Ophthalmol 98: 813-825. https://doi.org/10.1136/bjophthalmol-2013-304019

4. Joo K, Park SJ, Choi Y et al (2017) Role of the Fc region in the vitreous half-life of anti-VEGF drugs. Invest Ophthalmol Vis Sci 58:4261-4267. https://doi.org/10.1167/iovs.17-21813

5. del Amo EM, Rimpelä A-K, Heikkinen E et al (2017) Pharmacokinetic aspects of retinal drug delivery. Prog Retin Eye Res 57:134-185. https://doi.org/10.1016/j.preteyeres.2016.12.001

6. Deissler HL, Lang GK, Lang GE (2014) Capacity of aflibercept to counteract VEGF-stimulated abnormal behavior of retinal microvascular endothelial cells. Exp Eye Res 122:20-31. https://doi.org/ 10.1016/j.exer.2014.02.024

7. Deissler HL, Lang GK, Lang GE (2017) Neonatal Fc receptor FcRn is involved in intracellular transport of the Fc fusion protein aflibercept and its transition through retinal endothelial cells. Exp Eye Res 154:39-46. https://doi.org/10.1016/j.exer.2016.11.007

8. Dithmer M, Hattermann K, Pomarius P et al (2016) The role of Fcreceptors in the uptake and transport of therapeutic antibodies in the retinal pigment epithelium. Exp Eye Res 145:187-205. https://doi. org/10.1016/j.exer.2015.12.013

9. Junghans RP, Anderson CL (1996) The protection receptor for IgG catabolism is the $\beta_{2}$-microglobulin-containing neonatal intestinal transport receptor. Proc Natl Acad Sci U S A 93:5512-5516

10. Kim H, Fariss RN, Zhang C et al (2008) Mapping of the neonatal Fc receptor in the rodent eye. Invest Ophthalmol Vis Sci 49:20252029. https://doi.org/10.1167/iovs.07-0871

11. Powner MB, McKenzie JA, Christianson GJ et al (2014) Expression of neonatal $\mathrm{Fc}$ receptor in the eye. Invest Ophthalmol Vis Sci 55:1607-1615. https://doi.org/10.1167/iovs.13-12574

12. Deissler HL, Lang GK, Lang GE (2016) Internalization of bevacizumab by retinal endothelial cells and its intracellular fate: evidence for an involvement of the neonatal Fc receptor. Exp Eye Res 143:49-59. https://doi.org/10.1016/j.exer.2015.10.007

13. Ober RJ, Martinez C, Vaccaro C et al (2004) Visualizing the site and dynamics of IgG salvage by the MHC class I-related receptor, FcRn. J Immunol 172:2021-2029. https://doi.org/10.4049/ jimmunol.172.4.2021

14. Ward ES, Zhou J, Ghetie V, Ober RJ (2003) Evidence to support the cellular mechanism involved in serum IgG homeostasis in humans. Int Immunol 15:187-195

15. Ward ES, Martinez C, Vaccaro C et al (2005) From sorting endosomes to exocytosis: association of Rab4 and Rab11 GTPases with the Fc receptor, FcRn, during recycling. Mol Biol Cell 16:2028-2038. https://doi.org/10.1091/mbc.E04-08-0735

16. Ober RJ, Martinez C, Lai X et al (2004) Exocytosis of IgG as mediated by the receptor FcRn: an analysis at the single-molecule level. Proc Natl Acad Sci U S A 100:11076-11081. https://doi.org/ 10.1073/pnas.0402970101

17. Hasanagic M, Waheed A, Eissenberg JC (2015) Different pathways to the lysosome: sorting out alternatives. Int Rev Cell Mol Biol 320: 75-101. https://doi.org/10.1016/bs.ircmb.2015.07.008

18. Suzuki T, Ishii-Watabe A, Tada M et al (2010) Importance of neonatal FcR in regulating the serum half-life of therapeutic proteins containing the $\mathrm{Fc}$ domain of human IgG1: a comparative study of the affinity of monoclonal antibodies and Fc-fusion proteins to human neonatal FcR. J Immunol 184:1968-1976. https://doi.org/10. 04049/jimmunol.0903296

19. Christoforidis JB, Briley K, Binzel K et al (2017) Systemic biodistribution and intravitreal pharmacokinetic properties of bevacizumab, ranibizumab, and aflibercept in a nonhuman primate model. Invest Ophthalmol Vis Sci 58:5636-5645. https://doi.org/ 10.1167/iovs.17-22431

20. Kacskovics I, Wu Z, Simister NE et al (2000) Cloning and characterization of the bovine MHC class I-like Fc receptor. J Immunol 164:1889-1897

21. Deissler H, Deissler H, Lang GK, Lang GE (2005) Generation and characterization of iBREC: novel hTERT-immortalized bovine retinal endothelial cells. Int J Mol Med 15:65-70. https://doi.org/10. 3892/ijmm.16.1.65

22. Ober RJ, Radu CG, Ghetie V, Ward ES (2001) Differences in promiscuity for antibody-FcRn interactions across species: implications for therapeutic antibodies. Int Immunol 13:1551-1559. https://doi.org/10.1093/intimm/13.12.1551

23. Kacskovics I, Kis Z, Mayer B et al (2006) FcRn mediates elongated serum half-life of human IgG in cattle. Int Immunol 18:525-536. https://doi.org/10.1093/intimm/dxh393

24. Cui D, Zhang L, Li J et al (2014) Bovine FcRn-mediated human immunoglobulin $\mathrm{G}$ transfer across the milk-blood barrier in transgenic mice. PLoS One 9:e115972. https://doi.org/10.1371/journal. pone. 0115972

25. Presta LG, Chen H, O'Connor SJ et al (1997) Humanization of an anti-vascular endothelial growth factor monoclonal antibody for the therapy of solid tumors and other disorders. Cancer Res 57:45934599

26. Yu DY, Yu PK, Cringle SJ et al (2014) Functional and morphological characteristics of the retinal and choroidal vasculature. Prog Retin Eye Res 40:53-93. https://doi.org/10.1016/j.preteyeres. 2014.02.001

27. Deissler HL, Lang GK, Lang GE (2017) Inhibition of single routes of intracellular signaling is not sufficient to neutralize the biphasic disturbance of a retinal endothelial cell barrier induced by VEGFA $_{165}$. Cell Physiol Biochem 42:1493-1513. https://doi.org/10. $1159 / 000479213$

28. Hiwasa T, Sawada T, Sakiyama S (1990) Cysteine proteinase inhibitors and ras gene products share the same biological activities including transforming activity toward NIH3T3 mouse fibroblasts and the differentiation-inducing activity toward $\mathrm{PC} 12$ rat pheochromocytoma cells. Carcinogenesis 11:75-80

29. Inoue S, Bar-Nun S, Roitelman J, Simoni RD (1991) Inhibition of degradation of 3-hydroxy-3-methylglutaryl-coenzyme A reductase in vivo by cysteine protease inhibitors. J Biol Chem 266:1331113317

30. Mroczkiewicz M, Winkler K, Nowis D et al (2010) Studies of the synthesis of all stereoisomers of MG-132 proteasome inhibitors in the tumor targeting approach. J Med Chem 53:1509-1518. https:// doi.org/10.1021/jm901619n

31. McCarthy KM, Yoong Y, Simister NE (2000) Bidirectional transcytosis of IgG by the rat neonatal $\mathrm{Fc}$ receptor expressed in a rat kidney cell line: a system to study protein transport across epithelia. J Cell Sci 113:1277-1285

32. He W, Ladinsky MS, Huey-Tubman K et al (2008) FcRn-mediated antibody transport across epithelial cells revealed by electron tomography. Nature 455:542-546

33. Granger E, McNee G, Allan V, Woodman P (2014) The role of the cytoskeleton and molecular motors in endosomal dynamics. Sem Cell Dev Biol 31:20-29. https://doi.org/10.1016/j. semcdb.2014.04.011

34. Ng EL, Gan BQ, Ng F, Tang BL (2012) Rab GTPases regulating receptor trafficking at the late endosome-lysosome membranes. Cell Biochem Funct 30:515-523. https://doi.org/10.1002/cbf.2827 
35. D'Hooghe L, Chalmers AD, Heywood S, Whitley P (2017) Cell surface dynamics and cellular distribution of endogenous FcRn. PLoS One 12:e0182695. https://doi.org/10.1371/journal.pone. 0182695

36. Gan Z, Ram S, Vaccaro C et al (2009) Analyses of the recycling receptor, FcRn, in live cells reveal novel pathways for lysosomal delivery. Traffic 10:600-614. https://doi.org/10.1111/j.1600-0854. 2009.00887

37. Deissler HL, Deissler H, Lang GE (2012) Actions of bevacizumab and ranibizumab on microvascular retinal endothelial cells: similarities and differences. Br J Ophthalmol 96:1023-1028. https://doi. org/10.1136/bjophthalmol-2012-301677

38. Heiduschka P, Fietz H, Hofmeister S et al (2007) Penetration of bevacizumab through the retina after intravitreal injection in the monkey. Invest Ophthalmol Vis Sci 48:2814-2823
39. Kim H, Robinson SB, Csaky KG (2009) FcRn receptor-mediated pharmacokinetics of therapeutic IgG in the eye. Mol Vis 15:28032812

40. Schraermeyer U, Julien S (2012) Formation of immune complexes and thrombotic microangiopathy after intravitreal injection of bevacizumab in the primate eye. Graefes Arch Clin Exp Ophthalmol 250:1303-1313. https://doi.org/10.1007/s00417-0122055-z

41. Taubitz T, Steinbrenner LP, Tschulakow AV et al (2016) Effects of intravitreally injected Fc fragment on rat eyes. Graefes Arch Clin Exp Ophthalmol 254:2401-2409. https://doi.org/10.1007/s00417016-3511-y 